\title{
Dynamics of grating formation in photovoltaic media
}

\author{
Claire Gu and John Hong \\ Rockwell International Science Center, Thousand Oaks, California 91360
}

Hsin-Yu Li and Demetri Psaltis

California Institute of Technology, Pasadena, California 91125

Pochi Yeh

Rockwell International Science Center, Thousand Oaks, California 91360

(Received 11 June 1990; accepted for publication 16 October 1990)

The Kukhtarev equations are solved taking into account the photovoltaic effect and different boundary conditions. In the case of open circuit, the voltage across the crystal is found to vary with a time scale similar to the photorefractive time constant. This effect explains the dynamic behavior observed experimentally.

\section{INTRODUCTION}

Photorefractive crystals have become increasingly important as candidate media for the realization of many optical information processing systems including mass memory, ${ }^{1}$ neural network implementations, ${ }^{2-4}$ and signal processors. $^{5-7}$ Photorefraction in electro-optic crystals is well understood in terms of the band transport model ${ }^{8}$ which describes it in terms of the following processes: (1) the photo-excitation of charged carriers from impurities by a nonuniform intensity pattern, (2) charge transport mechanisms (diffusion, drift, photovoltaic) which move these charges in the conduction band to empty trap sites where they are captured, creating a nonuniform spacecharge field, and ( 3 ) nonuniform modulation of the index of refraction via the electro-optic effect.

Among the various transport mechanisms, the photovoltaic effect, in which a short-circuit current (or, equivalently, an open-circuit voltage) is generated by illumination of the crystal with light, has been observed only in a few ferroelectric crystals such as $\mathrm{LiNbO}_{3}$ and $\mathrm{KNbO}_{3}$. Although the photovoltaic effect is accounted for in the Kukhtarev equations, ${ }^{8}$ a transient theory that includes the effects due to the boundary conditions (e.g., open- or short-circuited crystal) has not yet been formulated. $\mathrm{LiNbO}_{3}$ which can be used for long-term memory storage applications due to its low dark conductivity, exhibits a particularly large photovoltaic effect, ${ }^{9}$ and such a theory may prove to be useful for further application developments. In this paper, we solve the Kukhtarev equations taking into account various possible boundary conditions to describe the growth and decay of holograms in photovoltaic media. We then give theoretical plots describing the transient behavior of holograms using published values of parameters for $\mathrm{LiNbO}_{3}$. The experimental results, showing different dynamic behaviors during grating formation for different boundary conditions, are then compared with the theoretical calculation. Both experimental result and theoretical simulation show the typical behavior of grating formation which does not occur in nonphotovoltaic media, i.e., when the writing starts from an open-circuited crystal without nonuniform charge distribution, the diffraction ef- ficiency increases to a maximum value then decreases to reach its steady state.

\section{FORMATION OF GRATINGS WITH APPLIED FIELD}

The dynamic behavior of the photorefractive effect is described by the following set of nonlinear coupled equations:

$$
\begin{aligned}
& \frac{\partial N_{D}^{+}}{\partial t}=\left(N_{D}-N_{D}^{+}\right)(s I+\beta)-\gamma_{R} N_{D}^{+} n, \\
& \frac{\partial n}{\partial t}=\frac{\partial N_{D}^{+}}{\partial t}+\frac{1}{e} \frac{\partial J}{\partial x} \\
& J=e \mu n E+k_{B} T \mu \frac{\partial n}{\partial x}+p\left(N_{D}-N_{D}^{+}\right) I, \\
& \epsilon \frac{\partial E}{\partial x}=e\left(N_{D}^{+}-n-N_{A}\right),
\end{aligned}
$$

where $N_{D}, N_{D}^{+}, n, N_{A}$ are densities of traps, ionized traps, charged carriers (electrons in the conduction band) and compensative acceptors, respectively, $I$ is the optical intensity in the crystal, $\beta$ is the thermal generation rate, $s$ is the photoexcitation cross section, $\mu$ is the carrier mobility, $e$ is the electronic charge, $\gamma_{R}$ is the carrier recombination rate constant, $\epsilon$ is the dielectric constant of the crystal, $E$ is the total electric field inside the crystal, and $p$ is the photovoltaic constant. The photovoltaic term in Eq. (3) depends on the nonionized trap density because the photovoltaic current is proportional to the photoabsorption constant which in turn depends on the nonionized trap density. In the special case of $N_{D}^{+} \ll N_{D}$, we can write this term as a constant times the intensity and is equivalent to eliminating all the terms containing density ratios $N_{A} / N_{D}$ in the final results. In this paper, we will include the $\left(N_{D}-N_{D}^{+}\right)$term explicitly. Following the standard procedure of linearization, which is legitimate for small modulation depth, we assume the following forms for the various physical quantities:

$$
\begin{aligned}
& N_{D}^{+}=N_{D 0}^{+}+\delta N_{D}^{+}, \\
& n=n_{0}+\delta n,
\end{aligned}
$$




$$
\begin{aligned}
& J=J_{0}+\delta J, \\
& E=E_{0}+\delta E,
\end{aligned}
$$

and then solve the coupled equations (1)-(4) for the zeroand first-order terms with an intensity given by

$$
I=I_{0}(1+m \cos K x),
$$

where $m$ is the modulation depth of the grating, and $K$ is the grating wave vector. The small modulation depth requires that $m<1$. Notice that when $m=0, E=E_{0}$. In other words, $E_{0}$ is the dc component of the spatial Fourier transform of $E . N_{D 0}^{+}$and $n_{0}$ change very rapidly towards their steady-state values, compared to the rate at which the spatially varying components change. ${ }^{10}$ Therefore, we solve the set of equations (1)-(4) by first calculating the stcady-statc valucs for $N_{D 0}^{+}$and $n_{0}$ and then using these as constants to solve for the temporal evolution of $\delta E$. In addition, we make the following assumptions: (1) $\beta$ $<s I_{0}$, (2) $n_{0} \ll N_{A}$, and (3) $s I_{0} \ll \gamma_{R} N_{A}$.

Under these conditions, the zero-order terms can be obtained as

$$
\begin{aligned}
& N_{D 0}^{+}=N_{A}, \\
& n_{0}=\frac{\left(N_{D}-N_{A}\right) s I_{0}}{\gamma_{R} N_{A}} .
\end{aligned}
$$

In order to proceed with the calculations of the first-order perturbation terms in Eqs. (5)-(8), we need to apply the appropriate boundary conditions so that the zero-order electric field $E_{0}$ can be determined. In this section, we examine the case of a constant applied field so that the term $E_{0}$ in Eq. (8) is equal to the externally applied electric field.

Substituting these zero-order terms along with Eqs. (5) $-(9)$ into the linearized Kukhtarev equations, we obtain a set of linear partial differential equations with constant coefficients. The equations can then be solved and the solution for the space-charge field is given by

$$
\begin{aligned}
\delta E= & m E_{\mathrm{sc}}[-\cos (K x+\psi)+\exp (-t / \tau) \cos (K x \\
& \left.\left.+\omega_{g} t+\psi\right)\right],
\end{aligned}
$$

where the amplitude $E_{\mathrm{sc}}$ is given by

$$
E_{\mathrm{sc}}=E_{q}\left(\frac{\left(E_{0}-E_{0 \mathrm{ph}}\right)^{2}+E_{D}^{2}}{\left[E_{0}-\left(N_{A} / N_{D}\right) E_{0 \mathrm{ph}}\right]^{2}+\left(E_{D}+E_{q}\right)^{2}}\right)^{1 / 2},
$$

and the phase of the grating is given by

$\tan \psi$

$$
=\frac{\left(E_{D}+E_{q}\right) E_{D}+\left[E_{0}-\left(N_{A} / N_{D}\right) E_{0 \mathrm{ph}}\right]\left(E_{0}-E_{0 \mathrm{ph}}\right)}{\left(E_{0}-E_{0 \mathrm{ph}}\right) E_{q}-E_{0 \mathrm{ph}} E_{D}\left[\left(N_{D}-N_{A}\right) / N_{D}\right]} .
$$

In arriving at these results, we have made use of the following expressions, in respective order, for the time constant, oscillatory frequency, dielectric relaxation time, diffusion field, saturation field, drift field, and photovoltaic field:

$$
\begin{aligned}
& \tau=\tau_{d i} \frac{\left[1+\left(E_{D} / E_{\mu}\right)\right]^{2}+\left(E_{0} / E_{\mu}\right)^{2}}{\left[1+\left(E_{D} / E_{q}\right)\right]\left[1+\left(E_{D} / E_{\mu}\right)\right]+\left(E_{0} / E_{\mu}\right)\left\{\left[E_{0}-\left(N_{A} / N_{D}\right) E_{0 \mathrm{ph}}\right] / E_{q}\right\}}, \\
& \omega_{g}=\frac{1}{\tau_{d i}} \frac{\left(E_{0} / E_{q}\right)-\left(E_{0} / E_{\mu}\right)-\left(N_{A} E_{0 \mathrm{ph}} / N_{D} E_{q}\right)\left[1+\left(E_{D} / E_{\mu}\right)\right]}{\left[1+\left(E_{D} / E_{\mu}\right)\right]^{2}+\left(E_{0} / E_{\mu}\right)^{2}},
\end{aligned}
$$

$$
\begin{aligned}
& \tau_{d i}=\epsilon / e \mu n_{0}, \\
& E_{D}=k_{B} T K / e, \\
& E_{q}=\frac{e N_{A}\left(N_{D}-N_{A}\right)}{\epsilon K N_{D}}, \\
& E_{\mu}=\gamma_{R} N_{A} / \mu K, \\
& E_{0 \mathrm{ph}}=\left(p \gamma_{R} N_{A} / e \mu s\right) .
\end{aligned}
$$

Several qualitative results need to be noted here. First, the photovoltaic effect cannot bc thought of as an equivalent applied field since the above expressions for the phase, time constant, and oscillatory frequency of the grating do not depend on a single linear combination of $E_{0}$ and $E_{0 \mathrm{ph}}$. The oscillatory frequency $\omega_{g}$ gives rise to ringing during the formation of the grating even in the absence of an external field, according to Eq. (16). In the limiting case of no photovoltaic effect (i.e., $p=0$ ), these solutions reduce to previous results. ${ }^{11}$

\section{GRATING FORMATION UNDER OPEN-CIRCUIT BOUNDARY CONDITION}

The experimental configuration most widely used is the open-circuit condition. In this case, photovoltaic crystals will develop an open-circuit voltage upon illumination whose time scale is similar to the photorefractive time constant. We need to evaluate the growth of the dc field $E_{0}(t)$, now a function of time, before we calculate the space-charge field.

The dc field $E_{0}(t)$ can be obtained by introducing a surface charge density $\sigma(t)$ at the ends of the crystal ${ }^{7}$ as illustrated in Fig. 1(a). The use of a surface charge distribution is justified by experimentally observing the macroscopic charge distribution that results when we uniformly 

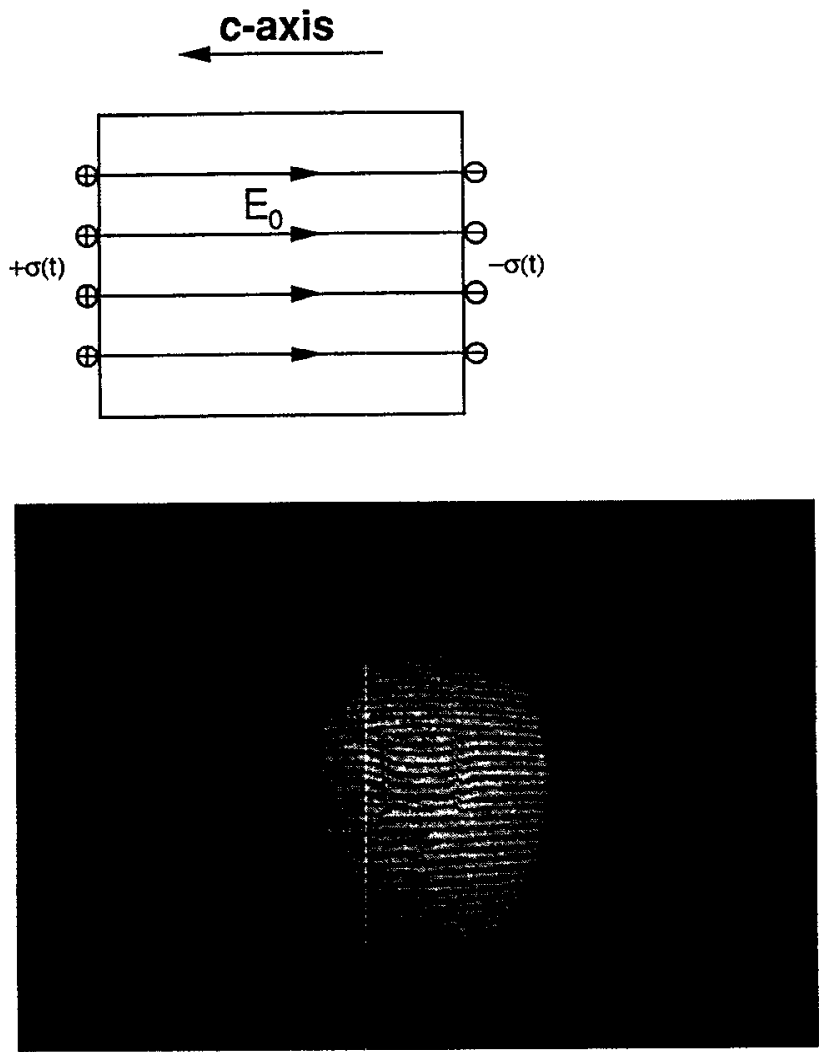

FIG. 1. (a) Open-circuit crystal (shows surface charge). (b) Interference fringes showing the index change of a partially illuminated crystal. The $c$ axis is parallel to the fringes, and the probe beam is extraordinarily polarized.

illuminate a portion of the crystal and examine the index change with an interferometer. Figure 1(b) shows the result of such an experiment. We notice that within the illuminated region the index change is uniform, indicating that the electric field is a constant. At the edges of the illuminated region, the refractive index changes rapidly within a thin layer of the crystal. Therefore, we approximate this charge distribution by a surface charge density. The surface charge $\sigma(t)$ is related in the zero-order current density and field, $J_{0}$ and $E_{0}$, by

$$
J_{0}=-\frac{d \sigma}{d t}
$$

and

$$
E_{0}=\sigma / \epsilon
$$

Again making use of the approximation that the carrier density equilibrates rapidly, we obtain

$$
E_{0}(t)=E_{0 \mathrm{ph}}\left[1-\exp \left(-t / \tau_{d i}\right)\right],
$$

where $E_{0 \mathrm{ph}}$ and $\tau_{d i}$ are given by Eqs. (21) and (17), respectively. Comparing Eq. (17) with Eq. (15), we see that the time constant for $E_{0}$ and that for $\delta E$ have the same order of magnitude. Therefore, we cannot assume that the dc field is approximately a constant during grating formation.
Because of this time variation in the zero-order field, the linearized Kukhtarev equations now have time-varying constants. We write the space-charge field as

$$
\delta E(t)=E_{1}(t) \exp (i K x)+\text { c.c. . }
$$

Substituting the above in the Kukhtarev equations and neglecting terms containing $d^{2} E_{1} / d t^{2}$, we arrive at the following differential equation for $E_{1}$ :

$$
\frac{d E_{1}}{d t}=-\frac{1}{\tau_{d i}}\left[a(t) E_{1}+b(t) E_{q}\right],
$$

where the time-varying constants are

$$
\begin{aligned}
& a(t)=\frac{1+\left(E_{D} / E_{q}\right)-i\left\{E_{0 \mathrm{ph}}\left[1-\exp \left(-t / \tau_{d i}\right)\right] / E_{q}\right\}}{1+\left(E_{D} / E_{\mu}\right)-i\left\{E_{0 \mathrm{ph}}\left[1-\exp \left(-t / \tau_{d i}\right)\right] / E_{\mu}\right\}}, \\
& b(t)=\frac{(m / 2)\left[-\left(E_{0 \mathrm{ph}} / E_{q}\right) \exp \left(-t / \tau_{d i}\right)+i\left(E_{D} / E_{q}\right)\right]}{1+\left(E_{D} / E_{\mu}\right)-i\left\{E_{0 \mathrm{ph}}\left[1-\exp \left(-t / \tau_{d i}\right)\right] / E_{\mu}\right\}} .
\end{aligned}
$$

In deriving the above expressions, we have assumed $N_{A}$ $\ll N_{D}$ for simplicity.

Before discussing the theoretical solution, we first present experimental results showing the striking differences in the diffraction efficiency during hologram growth for two different boundary conditions: (1) open circuit, (2) steady voltage. The open-circuit boundary condition is most easily achieved, as shown in Fig. 2(a), by writing a hologram whose overall transverse dimensions are smaller than the crystal dimensions (this is not really necessary but most easily achievable experimentally), so that the surface charge introduced earlier actually develops within the bulk of the crystal at the boundary between the illuminated and dark regions. After this experiment is performed, the hologram is carefully erased with a third beam which is not Bragg-matched to the written grating but illuminates only (approximately) the region where the grating exists, as shown in Fig. 2(b). The grating is thus erased but the open-circuit electric field remains across the region where the hologram was written. Now, the same grating can be written again in the same area under new boundary conditions imposed by the steady de field developed in the previous exposure, provided the field remains.

The $\mathrm{LiNbO}_{3}$ crystal $(0.015 \%$ Fe doped; $18 \mathrm{~mm} \times 18$ $\mathrm{mm} \times 8 \mathrm{~mm}: \mathrm{xyz}$ ) was antireflection coated on the broad faces. The apparatus for writing holographic gratings and monitoring their diffraction efficiencies is shown in Fig. 3. An argon laser $(\lambda=0.5145 \mu \mathrm{m})$ supplied the two writing beams ( 76.45 and $1.47 \mathrm{~mW}, 0.9-1 \mathrm{~mm}$ radius) which were both ordinary polarized to minimize beam coupling effects during recording and directed to write gratings whose orientation is parallel to the crystal $c$ axis (i.e., grating planes perpendicular to the $c$ axis, external angle between the two beams: $\left.16^{\circ}\right)$. A He-Ne laser $(\lambda=0.633 \mu \mathrm{m})$ supplied the reading beam $(43 \mu \mathrm{W})$ which was extraordinary polarized to access the large $r_{33}$ electro-optic coefficient. After writing a grating and carefully adjusting the reading beam to satisfy the Bragg condition, the writing beam was shut off, and the crystal was translated to a new position where 


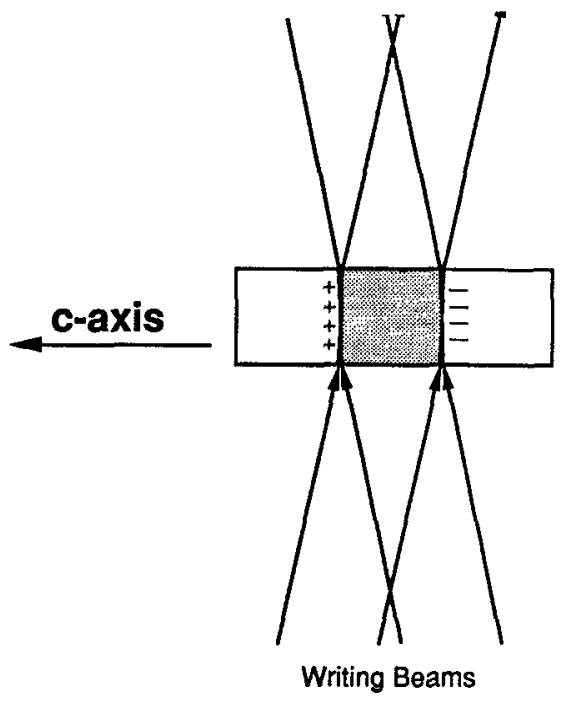

(a)

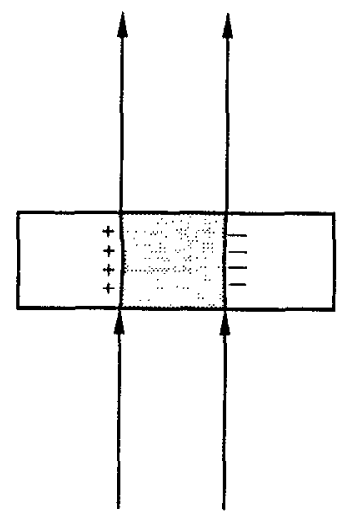

Erase Beam
FIG. 2. (a) Open-circuit boundary condition (beginning with a fresh crystal). (b) Grating erasure with non-Bragg-matched beam. gratings were not previously written ("fresh location") to perform the open-circuit boundary condition experiment. The writing beams were turned on and the growth of the hologram was monitored with the reading beam. The growth characteristics of the grating is shown in the lower trace in Fig. 4(a) where the grating is shown to peak initially and decrease to a smaller steady state value. Such a result was observed by earlier works ${ }^{12}$ but attributed to nonlinear beam coupling effects. In our experiment, the beam coupling was also monitored by recording the intensity of one of the writing beams (the pump, in two wave mixing language ${ }^{13,14}$ which is also shown in Fig. 4(a) (the upper trace). Apparently, there is very little pump depletion throughout the trace, hence beam coupling effects are not significant.

Next by optically erasing the previously written grating (power of the erasing beam: $525 \mathrm{~mW}$ ), an experiment to monitor grating development for the steady applied elec-

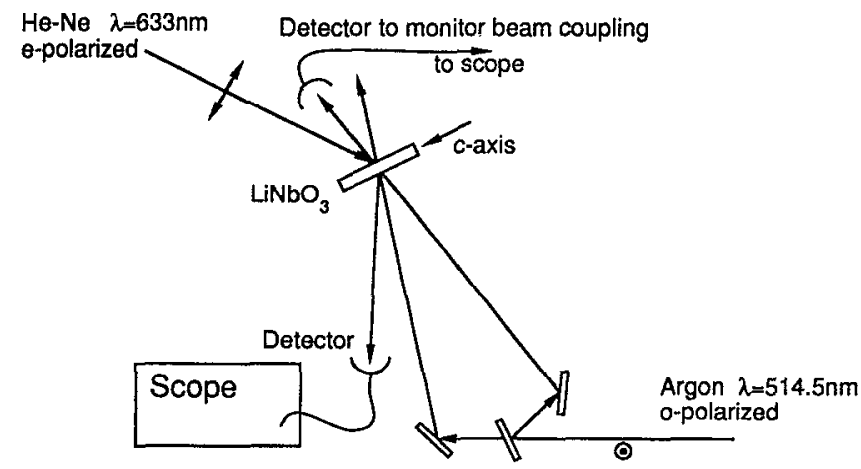

FIG. 3. Experimental apparatus for writing and monitoring gratings LiNbO ${ }_{3}$ crystal: $0.015 \%$ Fe doped, $18 \mathrm{~mm} \times 18 \mathrm{~mm} \times 8 \mathrm{~mm}: x y z$, antireflection coated on the broad faces. Writing beams: $\lambda=0.5145 \mu \mathrm{m}, 76.45$ $\mathrm{mW}$, and $1.47 \mathrm{~mW}$ (or $150 \mathrm{~mW}$ by control of a ND filter), $0.9-1 \mathrm{~mm}$ radius, ordinary polarized, external angle between the two beams $16^{\circ}$. Reading beam: $\lambda=0.633 \mu \mathrm{m}, 43 \mu \mathrm{W}$, extraordinary polarized. tric field was set up (power of the two writing beams: 76.45 and $150 \mathrm{~mW}$ ); the steady applied electric field is, as discussed previously, the open-circuit field that is set up by the photovoltaic effect created in the last exposure. The

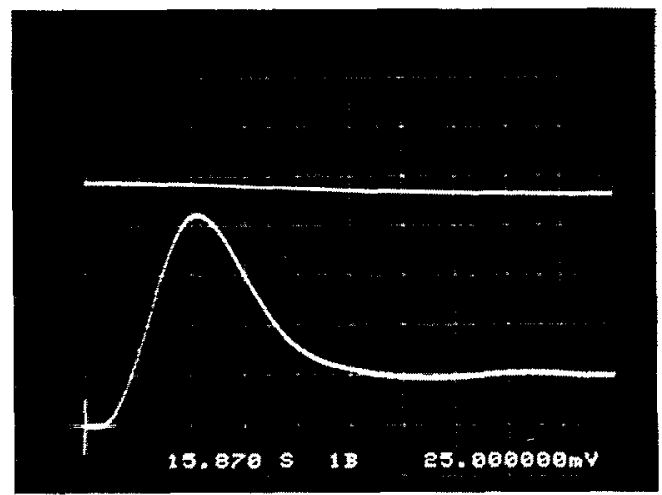

(a)

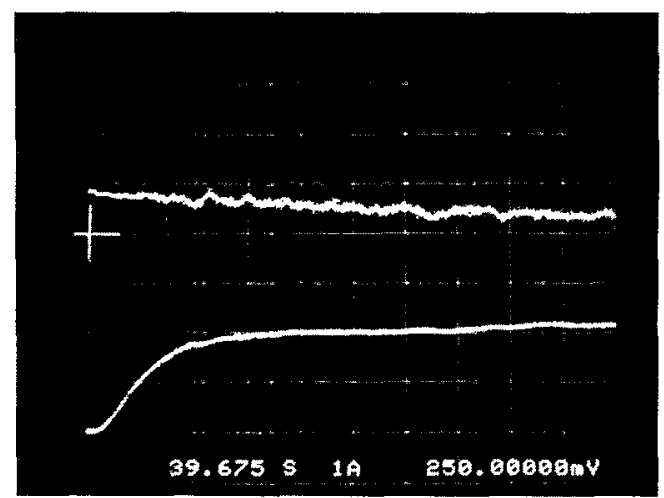

(b)

FIG. 4. Experimental curves for open-circuit hologram writing starting (a) from a fresh crystal (the scales are $15.870 \mathrm{~s} /$ div horizontally and 25 $\mathrm{mV} /$ div vertically), (b) after optical erasing (the scales are $39.675 \mathrm{~s} / \mathrm{div}$ horizontally and $250 \mathrm{mV} /$ div vertically). 


\section{Grating Formation}

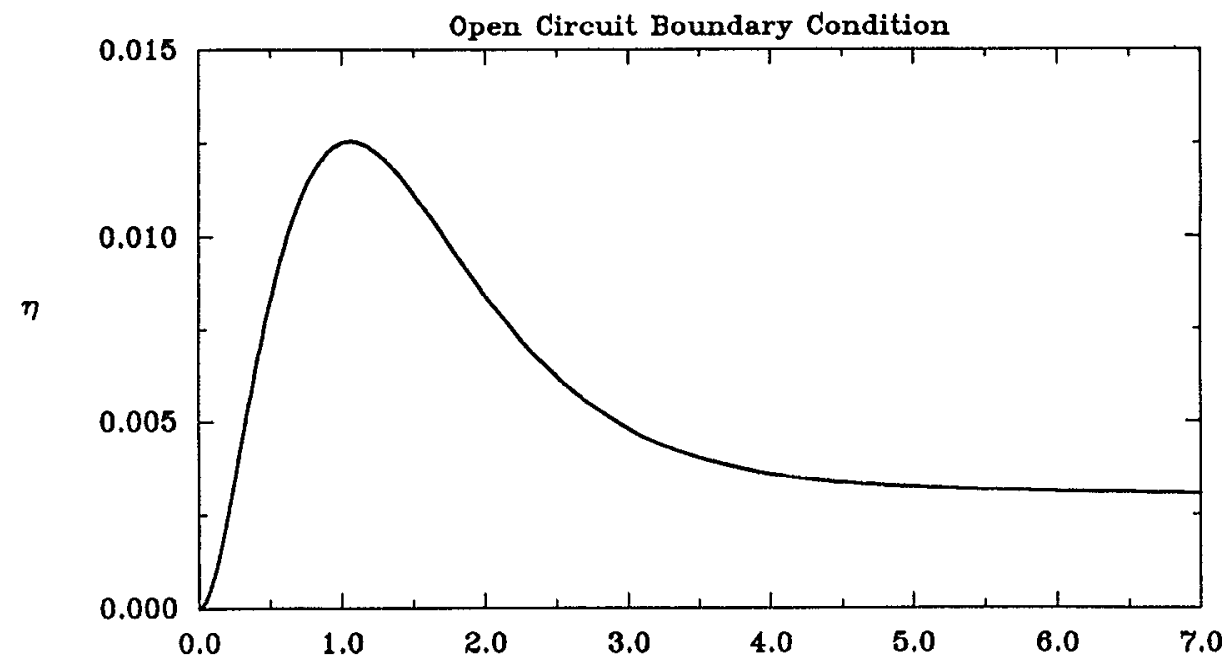

(a)

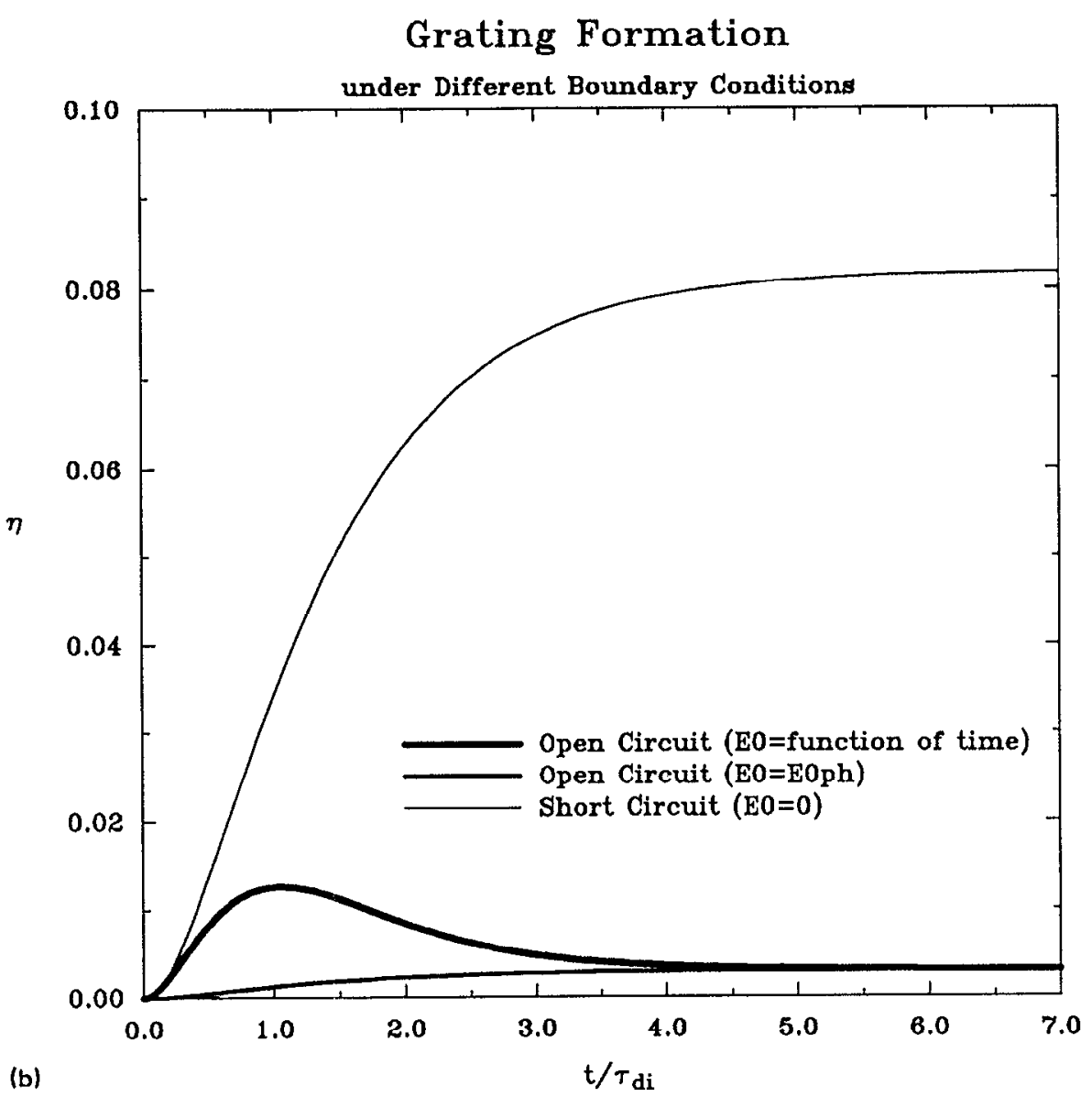

FIG. 5. Theoretical curves. (a) Fitting curve for Fig. 4(a). (b) Comparison of different boundary conditions. monitored diffraction efficiency is shown in Fig. 4(b), where, again, the beam coupling that was monitored was minimal. Note the strikingly different behavior in the two boundary conditions.

We now compare the observed results with our theoretical solutions. Eq. (26) is most easily solved numerically. In order to do this, we make use of several published parameters for Fe-doped $\mathrm{LiNbO}_{3}, \quad \mu \tau=1.6 \times 10^{-12}$ $\mathrm{cm}^{2} / \mathrm{V}$ and $\left|E_{0 \mathrm{ph}}\right|=4.5 \mathrm{KV} / \mathrm{cm}$ measured earlier for the particular crystal used in our experiment. ${ }^{7}$ Other parameters are estimated by fitting the experimental writing curve [Fig. 4(a)]. In Eqs. (18)-(21), the grating wave vector $K$ can be calculated from the external angle between the two writing beams $\left(16^{\circ}\right), T$ can be taken approximately as 
room temperature $(300 \mathrm{~K})$, and the unknown parameters are $N_{A}$ and $N_{D}$. We can adjust these two variables to fit the general shape of Fig. 4(a). The parameters we used for plotting Fig. 5(a) are as follows: $N_{A} \approx 2 \times 10^{16} / \mathrm{cm}^{3}$, $N_{D} \approx 4 \times 10^{17} / \mathrm{cm}^{3}, m=0.272, E_{D}=0.88 \mathrm{kV} / \mathrm{cm}$, $E_{\mu}=1.8 \times 10^{4} \mathrm{kV} / \mathrm{cm}, E_{q}=33 \mathrm{kV} / \mathrm{cm}$, and $\Lambda=1.85 \mu \mathrm{m}$. We now solve Eq. (26) using the fifth-order Runge-Kutta method ${ }^{15}$ with adaptive step size. Since there are more than one unknown parameter, we do not attempt to fit the experimental curve quantitatively, but the qualitative fit of the experimental result to theory is good.

In Fig. 5(b), we compare the diffraction efficiencies for different boundary conditions. It can be noticed, in the case of open circuit ( $E_{0}=$ function of time), the beginning of the grating formations is the same as that in the case of short circuit $\left(E_{0}=0\right)$. The steady-state diffraction efficiencies of the open-circuit case with $E_{0}$ a function of time and $E_{0}=E_{0 \mathrm{ph}}$ asymptotically converge. Between the beginning and the steady state, the diffraction efficiency reaches a maximum, then decreases to a constant. We can also notice that the steady-state diffraction efficiency of the shortcircuit case is higher than that of the open-circuit case.

\section{CONCLUSION}

We have theoretically explained the unique dynamic behavior of grating formation in photovoltaic media taking into account the time variation of the spatially uniform field across the crystal. Due to the photovoltaic effect, the dynamics of grating formation depends on the initial con- dition as well as the boundary condition. This effect causes a problem when we attempt to find an exposure procedure for the recording of multiple holograms. The results in this paper suggest that one simple solution might be to short circuit the crystal, since in this case the steady-state diffraction efficiency is higher than others and the initial condition remains the same for all exposures. However, more careful investigations of the boundary conditions that are established when a crystal is shorted may be necessary in order to accomplish the desired response in practice.

'F. Mok, M. Tackitt, and H. M. Stoll, Opt. Soc. Am. Tech. Dig. Ser. 12, 74 (1989)

${ }^{2}$ D. Psaltis, D. Brady, X.-G. Gu, and S. Lin, Nature 343, 325 (1990).

${ }^{3}$ L. S. Lee, H. M. Stoll, and M. C. Tackitt, Opt. Lett. 14, 162 (1989).

${ }^{4}$ B. H. Soffer, G. J. Dunning, Y. Owechko, and E. Marom, Opt. Lett. 11, 118 (1986).

${ }^{5}$ D. Psaltis and N. Farhat, Opt. Lett. 10, 98 (1985).

${ }^{\circ}$ J. Hong, S. Campbell, and P. Yeh, Opt. Soc. Am. Tech. Dig. Ser. 12, WJ3 (1989)

7J. Hong, S. Camphell, and P. Yeh, Proc. SPIE 1151, 56 (1989).

${ }^{8}$ N. V. Kukhtarev, V. B. Markov, S. G. Odulov, M. S. Soskin, and V. L. Vinetskii, Ferroelectrics 22, 949 (1979).

${ }^{9}$ A. M. Glass, Opt. Eng. 17, 470 (1978)

${ }^{10}$ G. C. Valley, Appl. Opt. 22, 3160 (1983).

"G. C. Valley and M. B. Klein, Opt. Eng. 22, 704 (1983).

${ }^{12}$ R. Magnusson and T. K. Gaylord, J. Appl. Phys. 47, 190 (1976).

${ }_{13}^{13}$ See, for example, P. Yeh, IEEE J. Quantum Electron. 25, 484 (1989).

${ }^{14}$ A. Yariv, Quantum Electronics, 3rd ed. (Wiley, New York, 1989), Chap. 19.

${ }^{15}$ See, for example, W. H. Press, B. P. Flannery, S. A. Teukolshy, and W. T. Vetterling, Numerical Recipes (Cambridge University Press, Cambridge, U.K., 1986). 